\title{
Rigorous Bounds on Greybody Factors: Scalar Emission of Negative Angular Momentum Modes from Myers-Perry Black Holes
}

\author{
Tritos Ngampitipan, Petarpa Boonserm, Auttakit Chatrabhuti, and Matt Visser
}

\begin{abstract}
When taking into account the quantum effects, a black hole can emit the so-called Hawking radiation. This Hawking radiation propagates in a curved spacetime due to the presence of a black hole. In this paper, the Myers-Perry black hole is considered, which is an uncharged, rotating black hole occurring in higher dimensions. Scalar Hawking radiation emitted from the Myers-Perry black hole is studied. The rigorous bounds on the greybody factors for massless scalar field of negative-angular-momentum modes are also derived.
\end{abstract}

Index Terms-Greybody factor, hawking radiation, myers-perry black hole, rigorous bound.

\section{INTRODUCTION}

The existence of black holes has been predicted by Einstein's general theory of relativity. The first solutions of the Einstein's field equation were discovered by Karl Schwarzschild. His solutions predicted the presence of Schwarzschild black holes, which are the uncharged, non-rotating black holes. The second type of black hole was obtained by solving the Einstein's field equation in conjunction with Maxwell's equation. This was done by Hans Reissner and Gunnar Nordström. Their solutions represented the Reissner- Nordström black holes, which are the charged, non-rotating black holes. The third set of solutions of the Einstein's field equation was discovered by Roy Kerr [1]. His solutions described the Kerr black holes, which are the uncharged, rotating black holes. The Kerr solutions were generalized to higher dimensions by Myers and Perry [2], [3] Their results led to the prediction of Myers-Perry black holes, which are the uncharged, rotating black holes in higher dimensions.

When studying the quantum effects of black holes, Stephen Hawking showed that black holes can emit thermal radiation which became known as Hawking radiation [4]. The curvature of spacetime due to the presence of a black hole acts as the gravitational potential barrier. The scattering of Hawking radiation from this potential can be viewed as

Manuscript received October 3, 2014; revised January 12, 2015.

T. Ngampitipan and A. Chatrabhuti is with the Particle Physics Research Laboratory, Department of Physics, Faculty of Science, Chulalongkorn University, Bangkok 10330, Thailand (e-mail: tritos.ngampitipan@gmail.com,dma3ac2@gmail.com)).

P. Boonserm is with the Department of Mathematics and Computer Science, Faculty of Science, Chulalongkorn University, Bangkok 10330, Thailand (e-mail: petarpa.boonserm@gmail.com).

M. Visser is with the School of Mathematics, Statistics, and Operations Research, Victoria University of Wellington, PO Box 600, Wellington, New Zealand (e-mail: matt.visser@msor.vuw.ac.nz). one-dimensional scattering problem in quantum mechanics. The term 'greybody factor' can be defined as the transmission probability.

In this paper, the rigorous bounds on the greybody factors for massless scalar field of negative-angular-momentum modes emitted from a Myers-Perry black hole will be derived.

\section{MYERS-PERRY SPACETIME}

The Myers-Perry spacetime can be described by the metric [2], [3], [5]

$$
\begin{aligned}
d s^{2}= & -d t^{2}+\frac{\Sigma}{\Delta} d r^{2}+\Sigma d \theta^{2}+\left(r^{2}+a^{2}\right) \sin ^{2} \theta d \varphi^{2} \\
& +\frac{\mu}{r^{n-1} \Sigma}\left(d t-a \sin ^{2} \theta d \varphi\right)^{2}+r^{2} \cos ^{2} \theta d \Omega_{n}^{2}
\end{aligned}
$$

where

$$
\Delta=r^{2}+a^{2}-\frac{\mu}{r^{n-1}} \text { and } \Sigma=r^{2}+a^{2} \cos ^{2} \theta
$$

Here $d \Omega_{n}^{2}$ is the metric on the unit $n$-sphere $S^{n}$ which is given by

$$
d \Omega_{n}^{2}=\left(\prod_{i=1}^{n-1} \sin ^{2} \theta_{i}\right) d \theta_{n}^{2}
$$

The solutions of $\Delta(r)=0$ provide the location of the black hole event horizons. In this paper, we focus on massless scalar field emitted from the Myers-Perry black hole. The equation of motion of this scalar field can be described by the Klein-Gordon equation

$$
\partial_{\mu}\left(\sqrt{-g} g^{\mu v} \partial_{v} \Phi\right)=0
$$

where

$$
\sqrt{-g}=(\Sigma \sin \theta) \times\left(r^{n} \cos ^{n} \theta\right) \times\left(\prod_{i=1}^{n-1} \sin ^{n-i} \theta_{i}\right)
$$

This Klein-Gordon equation governs how the scalar field $\Phi$ propagates in the Myers-Perry background. We use the separation of variables in this form 


$$
\begin{aligned}
\Phi(t, r, \theta, \varphi & \left., \theta_{1}, \ldots, \theta_{n}\right) \\
& =e^{-i \omega t+i m \varphi} \tilde{R}_{j \ell m}(r) S_{\ell m}(\theta) Y_{j n}\left(\theta_{1}, \ldots, \theta_{n}\right),
\end{aligned}
$$

where $S_{\ell m}(\theta) e^{i m \varphi}$ are the spheroidal harmonics and $Y_{j n}\left(\theta_{1}, \ldots, \theta_{n}\right)$ are the hyper-spherical harmonics. The spheroidal harmonics satisfy

$$
\begin{gathered}
\left\{\frac{1}{\sin \theta \cos ^{n} \theta} \frac{d}{d \theta}\left[\sin \theta \cos ^{n} \theta \frac{d}{d \theta}\right]-\left(\omega a \sin \theta-\frac{m}{\sin \theta}\right)^{2}\right. \\
\left.-\frac{j(j+n-1)}{\cos ^{2} \theta}+\lambda_{j \ell m}\right\} S_{\ell m}(\theta)=0
\end{gathered}
$$

while the hyper-spherical harmonics satisfy

$$
\Delta_{S^{n}} Y_{j n}\left(\theta_{1}, \ldots, \theta_{n}\right)+j(j+n-1) Y_{j n}\left(\theta_{1}, \ldots, \theta_{n}\right)=0,
$$

where $\Delta_{S^{n}}$ is the Laplacian. Then, the radial Teukolsky equation is obtained [6]-[8]

$$
\left[\frac{d^{2}}{d r_{*}^{2}}-U_{j \ell m}(r)\right] R_{j \ell m}(r)=0
$$

where the tortoise coordinate $r_{*}$ is defined by

$$
d r_{*}=\frac{r^{2}+a^{2}}{\Delta(r)} d r
$$

The relationship between the tortoise coordinate and the ordinary coordinate is plotted as shown in Fig. 1.

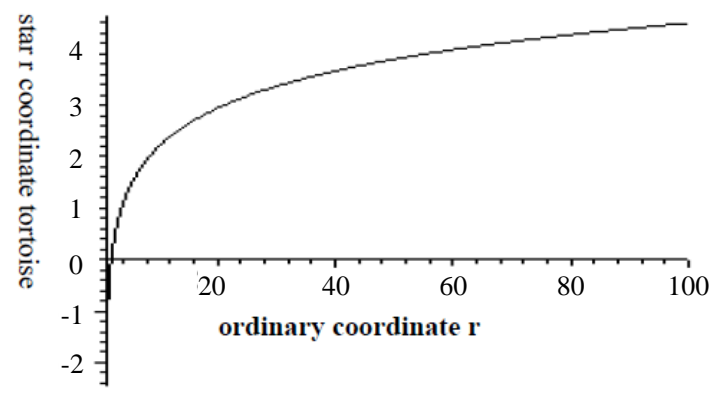

Fig. 1. Tortoise coordinate as a function of ordinary coordinate.

Here the Teukolsky potential $U_{j \ell m}(r)$ is given by [5]

$$
\begin{aligned}
U_{j \ell m}(r) & =\frac{\Delta(r)}{\left(r^{2}+a^{2}\right)^{2}}\left[\lambda_{j \ell m}+\frac{j(j+n-1) a^{2}}{r^{2}}+\frac{n(n-2) \Delta(r)}{4 r^{2}}\right. \\
& \left.+\frac{n \Delta^{\prime}(r)}{2 r}-\frac{3 r^{2} \Delta(r)}{\left(r^{2}+a^{2}\right)^{2}}+\frac{[r \Delta(r)]^{\prime}}{r^{2}+a^{2}}\right]-(\omega-m \varpi)^{2},(11)
\end{aligned}
$$

$$
\varpi=\frac{a}{r^{2}+a^{2}}
$$

This Teukolsky potential can be expressed in another form as

$$
U_{j \ell m}(r)=V_{j \ell m}(r)-(\omega-m \varpi)^{2},
$$

where

$$
\begin{gathered}
V_{j \ell m}(r)=\frac{\Delta(r)}{\left(r^{2}+a^{2}\right)^{2}}\left[\lambda_{j \ell m}+\frac{j(j+n-1) a^{2}}{r^{2}}+\frac{n(n-2) \Delta(r)}{4 r^{2}}\right. \\
\left.+\frac{n \Delta^{\prime}(r)}{2 r}-\frac{3 r^{2} \Delta(r)}{\left(r^{2}+a^{2}\right)^{2}}+\frac{[r \Delta(r)]^{\prime}}{r^{2}+a^{2}}\right]
\end{gathered}
$$

The potential $V_{j \ell m}(r)$ is plotted as shown in Fig. 2 for five and six dimensions which correspond to $n=1$ and $n=2$, respectively.

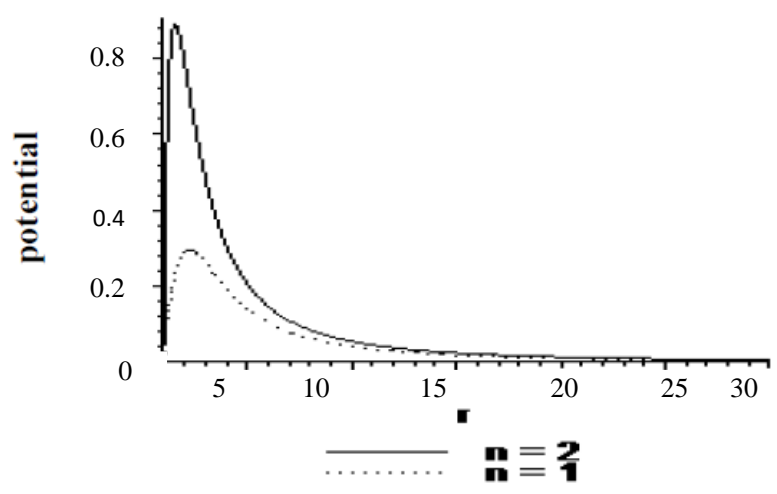

Fig. 2. The potential $V_{j \prime m}(r)$ for $n=1$ and $n=2$.

\section{RIgOROUS BOUNDS ON GREYBODY FACTORS}

We can model the scattering of the massless scalar field from the Teukolsky potential as one-dimensional scattering problem in quantum mechanics. The term 'greybody factor' in black hole systems can be defined as the 'transmission probability'. In general situations, finding exact greybody factors is difficult due to complicated potentials. Therefore, in this paper, some rigorous bounds will be placed on greybody factors. These bounds were first developed in [9]. Their further developments can be found in [10]-[13] and their applications can be found in [14]-[19]. For the radial Teukolsky equation in (9), the rigorous bounds on the greybody factors are given by

$$
T_{j \ell m} \geq \operatorname{sech}^{2}\left[\int_{-\infty}^{\infty} \frac{\left|\tilde{h}^{\prime}\left(r_{*}\right)\right|}{2 \tilde{h}\left(r_{*}\right)} d r_{*}+\int_{-\infty}^{\infty} \frac{\left|\tilde{U}_{j \ell m}\left(r_{*}\right)+\tilde{h}^{2}\left(r_{*}\right)\right|}{2 \tilde{h}\left(r_{*}\right)} d r_{*}\right]
$$

for any positive functions $h\left(r_{*}\right)$. In this paper, we choose

$$
\tilde{h}\left(r_{*}\right)=h(r)=\omega-m \varpi(r),
$$


where $m<0$. In this case, we obtain

$$
\tilde{h}^{\prime}\left(r_{*}\right)=\frac{2 \operatorname{mar} \Delta(r)}{\left(r^{2}+a^{2}\right)^{3}}>0 \text {. }
$$

Then, we obtain the first integral

$$
\int_{-\infty}^{\infty} \frac{\left|\tilde{h}^{\prime}\left(r_{*}\right)\right|}{2 \tilde{h}\left(r_{*}\right)} d r_{*}=\frac{1}{2} \ln \frac{\tilde{h}(\infty)}{\tilde{h}(-\infty)}=\frac{1}{2} \ln \left(1-m / m_{*}\right),
$$

where

$$
m_{*}=\frac{\omega}{\Omega_{H}}, \Omega_{H}=\frac{a}{a^{2}+r_{H}^{2}},
$$

and $r_{H}$ is the event horizon radius Since $\omega-m \Omega_{H}>$ $h(r)>\omega$, we have an inequality

$$
\int_{-\infty}^{\infty} \frac{\left|U_{j \ell m}+h^{2}(r)\right|}{2 h(r)} d r_{*}=\int_{-\infty}^{\infty} \frac{\left|V_{j \ell m}\right|}{2 h(r)} d r_{*}<\int_{-\infty}^{\infty} \frac{V_{j \ell m}}{2 \omega} d r_{*}
$$

Using (14), we can write

$$
\begin{aligned}
\int_{-\infty}^{\infty} \frac{V_{j \ell m<0}}{2 \omega} d r_{*}= & \frac{1}{2 \omega} \int_{-\infty}^{\infty} \frac{\Delta(r)}{\left(r^{2}+a^{2}\right)^{2}}\left[\lambda_{j \ell m<0}+\frac{j(j+n-1) a^{2}}{r^{2}}\right. \\
& +\frac{n(n-2) \Delta(r)}{4 r^{2}}+\frac{n \Delta^{\prime}(r)}{2 r} \\
& \left.-\frac{3 r^{2} \Delta(r)}{\left(r^{2}+a^{2}\right)^{2}}+\frac{[r \Delta(r)]^{\prime}}{r^{2}+a^{2}}\right] d r_{*}
\end{aligned}
$$

Using (10), we can change the variable $r_{*}$ to $r$

$$
\begin{aligned}
\int_{-\infty}^{\infty} \frac{V_{j \ell m<0}}{2 \omega} d r_{*}= & \frac{1}{2 \omega} \int_{r_{H}}^{\infty} \frac{\Delta(r)}{\left(r^{2}+a^{2}\right)^{2}}\left[\lambda_{j \ell m<0}+\frac{j(j+n-1) a^{2}}{r^{2}}\right. \\
& +\frac{n(n-2) \Delta(r)}{4 r^{2}}+\frac{n \Delta^{\prime}(r)}{2 r} \\
& \left.-\frac{3 r^{2} \Delta(r)}{\left(r^{2}+a^{2}\right)^{2}}+\frac{[r \Delta(r)]^{\prime}}{r^{2}+a^{2}}\right] \frac{r^{2}+a^{2}}{\Delta(r)} d r .
\end{aligned}
$$

The above equation can be simplified to

$$
\begin{aligned}
\int_{-\infty}^{\infty} \frac{V_{j \ell m<0}}{2 \omega} d r_{*}= & \frac{1}{2 \omega} \int_{r_{H}}^{\infty} \frac{1}{r^{2}+a^{2}}\left[\lambda_{j \ell m<0}+\frac{j(j+n-1) a^{2}}{r^{2}}\right. \\
& +\frac{n(n-2) \Delta(r)}{4 r^{2}}+\frac{n \Delta^{\prime}(r)}{2 r} \\
& \left.-\frac{3 r^{2} \Delta(r)}{\left(r^{2}+a^{2}\right)^{2}}+\frac{[r \Delta(r)]^{\prime}}{r^{2}+a^{2}}\right] d r .
\end{aligned}
$$

Therefore,

$$
T_{j \ell, m<0} \geq \operatorname{sech}^{2}\left[\frac{1}{2} \ln \left(1-m / m_{*}\right)+\frac{1}{2 \omega r_{H}} I_{j \ell, m<0}\right],
$$

where

$$
\begin{aligned}
I_{j \ell m<0}= & \frac{n(2 n-3)}{8}+j(j+n-1)+\frac{a^{2}}{4\left(r_{H}^{2}+a^{2}\right)} \\
& +\left(\frac{2 n+1}{2}-j(j+n-1)+\lambda_{j \ell m}(a \omega)\right) \frac{r_{H}}{a} \arctan \frac{a}{r_{H}} \\
& +\frac{n\left(r_{H}^{2}+a^{2}\right)}{8 r_{H}^{2}}{ }_{2} F_{1}\left(1, \frac{n+2}{2}, \frac{n+4}{2},-\frac{a^{2}}{r_{H}^{2}}\right) .
\end{aligned}
$$

Here the hypergeometric function ${ }_{2} F_{1}(a, b, c, z)$ is defined by

$$
{ }_{2} F_{1}(a, b, c, z)=\sum_{n=0}^{\infty} \frac{(a)_{n}(b)_{n}}{(c)_{n}} \frac{z^{n}}{n !} .
$$

The bounds on the greybody factors are plotted as shown in Fig. 3.

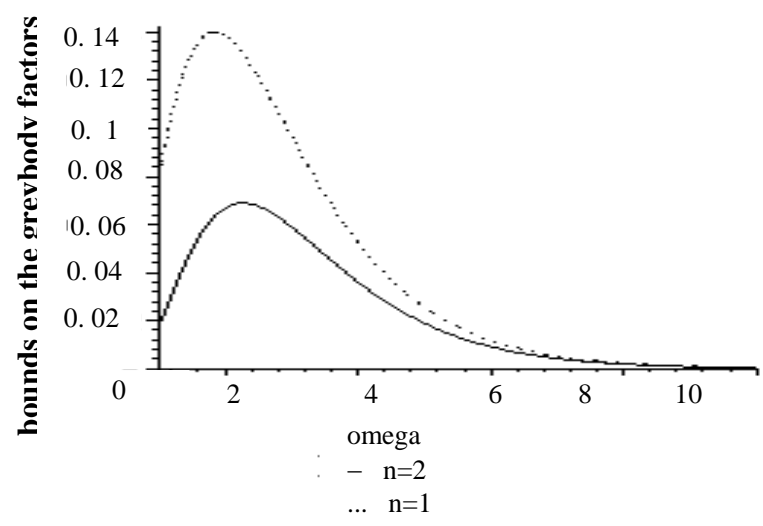

Fig. 3. The bounds on the greybody factors for $n=1$ and $n=2$.

In the limit $a \rightarrow 0$ and $n=j=0$, the quantity $I_{j \ell m<0}$ reduces to

$$
I_{j=0, \ell, m<0}=\frac{1}{2}+\lambda_{j=0, \ell, m<0},
$$

which is the result for the Schwarzschild black hole [14].

\section{CONCLUSION}

In this paper, the rigorous bounds on the greybody factors for massless scalar field of negative-angular-momentum modes emitted from the Myers-Perry black hole have been established. To obtain these bounds, the appropriate function $h\left(r_{*}\right)$ has been chosen. The number of dimensions of spacetime, the angular momentum of the black holes, and the mass of the black hole have been determined to have effects on these bounds. Note that for $n=0$, these bounds reduce to bounds for Kerr black holes. For outlook, we can choose other forms of $h(r *)$ in order to derive better bounds. 


\section{ACKNOWLEDGMENT}

This project was funded by Ratchadapisek Sompoch Endowment Fund, Chulalongkorn University (Sci-Super 2014-032), by a grant for the professional development of new academic staff from the Ratchadapisek Somphot Fund at Chulalongkorn University, by the Thailand Toray Science Foundation (TTSF), by the Thailand Research Fund (TRF), by the Office of the Higher Education Commission (OHEC), Faculty of Science, Chulalongkorn University (MRG5680171), and by the Research Strategic plan program (A1B1). AC was supported by the Thailand Toray Science Foundation (TTSF) and Thailand Excellence in Physics project (THEP). PB was additionally supported by a scholarship from the Royal Government of Thailand. TN was also additionally supported by a scholarship from the Development and Promotion of Science and Technology talent project (DPST). MV was supported by the Marsden Fund, and by a James Cook fellowship, both administered by the Royal Society of New Zealand.

\section{REFERENCES}

[1] R. P. Kerr, "Gravitational field of a spinning mass as an example of algebraically special metrics," Physical Review Letter, vol. 11, pp. 237-238, September 1963.

[2] R. C. Myers and M. J. Perry, "Black holes in higher dimensional space-times," Annals of Physics, vol. 172, pp. 304-347, December 1986.

[3] R. Emparan and H. S. Reall, "Black holes in higher dimensions," Living Reviews in Relativity, vol. 11, September 2008.

[4] S. W. Hawking, "Particle creation by black holes," Communications in Mathematical Physics, vol. 43, pp. 199-220, April 1975.

[5] T. Ngampitipan, P. Boonserm, A. Chatrabhuti, and M. Visser, "Spin-zero hawking radiation: Bounds on the zero-angular-momentum mode emission from Myers-Perry black holes," in Proc. the International Conference on Multi-Hazard Approaches to Civil Infrastructure Engineering, 2014.

[6] D. Ida, Y. Uchida, and Y. Morisawa, "The scalar perturbation of higher-dimensional rotating black holes," Physical Review D, vol. 67, April 2003.

[7] S. Creek, O. Efthimiou, P. Kanti, and K. Tamvakis, "Scalar emission in the bulk in a rotating black hole background," Physics Letter B, vol. 656, pp. 102-111, November 2007.

[8] S. Creek, O. Efthimiou, P. Kanti, and K. Tamvakis, "Greybody factors for brane scalar fields in a rotating black-hole background," Physical Review D, vol. 75, April 2007.

[9] M. Visser, "Some general bounds for 1-D scattering," Physical Review $A$, vol. 59, pp. 427-438, January 1999.

[10] P. Boonserm and M. Visser, "Transmission probabilities and the Miller-Good transformation," Journal of Physics A: Mathematical and Theoretical, vol. 42, January 2009.

[11] P. Boonserm and M. Visser, "Bounding the Bogoliubov coefficients," Annals of Physics, vol. 323, pp. 2779-2798, November 2008.

[12] P. Boonserm and M. Visser, "Analytic bounds on transmission probabilities,” Annals of Physics, vol. 325, pp. 1328-1339, April 2010.

[13] P. Boonserm and M. Visser, "Reformulating the equation as a Shabat-Zakharov system," Journal of Mathematical Physics, vol. 51, February 2010.

[14] P. Boonserm and M. Visser, "Bounding the greybody factors for Schwarzschild black holes," Physical Review D, vol. 78, November 2008 .
[15] T. Ngampitipan and P. Boonserm, "Bounding the greybody factors for non-rotating black holes," International Journal of Modern Physics D, vol. 22, June 2013

[16] T. Ngampitipan and P. Boonserm, "Bounding the greybody factors for the Reissner-Nordström black holes," Journal of Physics: Conference Series, vol. 435, May 2013.

[17] P. Boonserm, T. Ngampitipan, and M. Visser, "Regge-Wheeler equation, stability, and greybody factors for dirty black holes," Physical Review D, vol. 88, August 2013.

[18] P. Boonserm, T. Ngampitipan, and M. Visser, "Bounding the greybody factors for scalar field excitations of the Kerr-Newman spacetime," Journal of High Energy Physics, vol. 1403, p. 113, March 2014.

[19] P. Boonserm, A. Chatrabhuti, T. Ngampitipan, and M. Visser, "Greybody factors for Myers-Perry black holes," Journal of Mathematical Physics, vol. 55, November 2014.

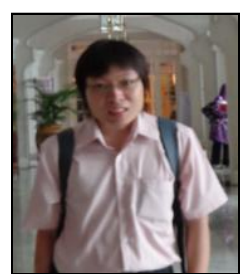

Tritos Ngampitipan received the B.Sc., M.Sc., and Ph.D. degrees in physics from Chulalongkorn University, Bangkok, Thailand, in 2008, 2011, and 2015, respectively.

$\mathrm{He}$ is a researcher at the Department of Physics, Faculty of Science, Chulalongkorn University, Bangkok, Thailand. His current research interests include quantum theory and general relativity.

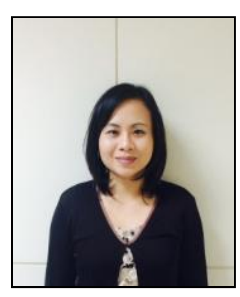

Petarpa Boonserm received the BSc., MSc., and Ph.D. degrees in mathematics from Victoria University of Wellington, Wellington, New Zealand, in 2004, 2006, and 2009, respectively.

She is an assistant professor at the Department of Mathematics and Computer Science, Faculty of Science, Chulalongkorn University, Bangkok, Thailand. Her current research interests include differential equations, applied mathematics, quantum theory, and general relativity.

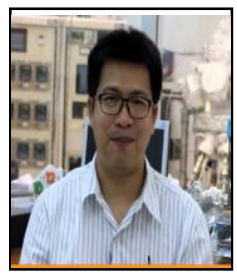

Auttakit Chatrabhuti received the Ph.D. degree from Durham University, Durham, United Kingdom, in 2002 .

$\mathrm{He}$ is an assistant professor at the Department of Physics, Faculty of Science, Chulalongkorn University, Bangkok, Thailand. His current research interests include quantum field theory, string theory, and supersymmetry.

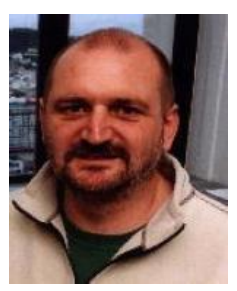

Matt Visser received the Ph.D. degree from University of California at Berkeley, California, USA. $\mathrm{He}$ is a professor at the School of Mathematics, Statistics, and Operations Research, Victoria University of Wellington, Wellington, New Zealand. $\mathrm{He}$ is best known for his contributions to the theory of traversable wormholes, chronology protection, and analogue spacetimes. His research interests include cosmology. general relativity, quantum field theory, and 